\title{
Anesthesia management in a patient with Situs inversus totalis: Case report
}

\author{
Abdullah Kahraman* \\ Department of Anestesiology, V an State Education and Research Hospital, Health Science University, Ministry of Health, Van, \\ Turkey
}

\begin{abstract}
Situs inversus totalis is an autosomal recessive congenital anomaly, seen at 1/7000-8000 ratio. It is quite a rare condition characterized by asymmetric placement of abdominal and thoracic organs. In our case, in the cause of acute abdomen, an emergency operation was planned for a twenty-one years old patient diagnosed with situs inversus. Based on a detailed examination and patient history, it was established that there were no additional diseases. The patient was taken to the operation room and was routinely monitored. After anesthesia induction, surgical laparoscopic surgery was applied and during the exploration situs inversus totalis was observed in the patient. Appendectomy was applied to the patient. The patient whose vitals were stable during the operation was extubated postoperatively and sent to the service. Situs inversus totalis is an isolated case, and doesn't carry additional pathology with it, however we would like to remind that it may collocate with heart, spine, and airway borne structural and functional malformations.
\end{abstract}

Key Words: Anesthesia management, appendectomy, general anesthesia, Situs inversus totalis

\section{Introduction}

Situs inversus totalis (SIT) is a rare anatomical condition characterized by asymmetrical placement of the thoracic and abdominal organs. Situs inversus totalis (SIT) occurs when embryonic midgut rotates $270^{\circ}$ clockwise instead of rotating $270^{\circ}$ counterclockwise. So all the thoracic and abdominal visceral organs are placed in symmetrical localization of the mid-line localization they normally are supposed to be. In other words, situs inversus is the mirror image of the normal (1). Situs inversus (SI) can be called as total when it contains thoracic and abdominal organ asymmetry at once and as partial when it contains the half (2). Situs inversus (SI), is an autosomal recessive congenital anomaly, and is seen at $1 / 5000-10000$ ratio. While SI may be seen alone, it may frequently associates with Kartagener Syndrome of which sinusitis and bronchiectasis are components or with ciliary dysfunction such as cystic fibrosis (2).

In this study, we wanted to remind that rarely seen SIT can occur as isolated or with diseases that are accompanied by especially heart, spine and airway borne structural and functional malformations.

\section{Case report}

A twenty-one one years old, $160 \mathrm{~cm}$ tall, $70 \mathrm{~kg}$ male patient in acute abdomen condition with severe abdominal pain, nausea and vomiting complaints. The patient history informed of situs inversus totalis. The abdominal ultrasound examination of the patient conformed to appendicitis. The other findings with the ultrasound were the liver and the gall bladder was placed on the left, while the spleen was placed on the right. As a result of these findings, the patient was scheduled for laparoscopic surgery for appendectomy. Before pre-medication the patient was taken into the operation room and routinely monitored. $500 \mathrm{ml}$ of $0.9 \%$ isotonic fluid was given to the patient via venous access. Heart rate 80 pulse/minute, blood pressure 125/60 $\mathrm{mmHg}$, peripheral oxygen saturation of the patient were measured as 97\%. $160 \mathrm{mg}$ propofol for intravenous anesthesia induction, 100 mcg fentanyl, $40 \mathrm{mg}$ rocuronium were used, intubation was performed with 7.0 numbered cuffed tube. During laparoscopic surgery, situs inversus totalis was confirmed. Liver and caecum were placed on the left side while spleen and stomach were on the right side.

Appendix was observed as hyperemic edematous and stretched. The patient underwent the process of 
appendectomy. The patient whose intraoperative vital signs were stable was extubated and sent to the service without any problems.

\section{Discussion}

Total Situs Inversus occurs when midgut rotates $270^{\circ}$ clockwise instead of rotating $270^{\circ}$ counter-clockwise in the embryonic period. So all the thoracic and abdominal visceral organs are placed in symmetrical localization of the mid-line localization they normally are supposed to be, in other words the organs are placed in mirror image. In this case, the appendix is located in lower left quadrant.

As appendix is in abnormal localization, it can be difficult to diagnose appendicitis in cases with situs inversus totalis. In these cases, due to late diagnosis complications such as abscess and perforation which may make the clinical course worse and make operation difficult. The most important ways to prevent these complications are detailed anamnesis, careful physical examination and making use of imaging methods. In physical examination, the right position of heart pulse, the significant intensity of the heart sounds in the right compared to the left side and palpability of the liver on the left suggest situs inversus. Direct graphies, ultrasound, barium x-ray imaging and abdominal tomography are radiological examinations that can help diagnosing situs inversus $(2,3)$.

Situs inversus is a condition characterized by anatomical positional incongruity, the frequency of which ranges between 1:7000-1:8000 in live births. Male/female ratio is $1 / 1$. Kartagener Syndrome develops in $15-25 \%$ of children with Situs inversus (4). These cases pursue normal lives unless accompanied with severe cardiac anomalies. There is an autosomal recessive genetic predisposition. It is a transposition of the abdomen and chest organs, which is in the form of an exact mirror image of these organs (5). While this condition can be isolated, it can sometimes be accompanied by other abnormalities. These are cardiovascular abnormalities such as; ASD, VSD, transposition of the great arteries, spinal anomalies such as; scoliosis, meningomiyelocel, spina bifida and intestinal malrotations. It can occur often as a component of Kartagener Syndrome and rarely can accompany syndromes such as; Carpenter syndrome (6), Jeune Syndrome (7), hypoplastic left heart syndrome, asplenia and polysplenia syndrome. Kartegener consists of Situs Inversus Totalis, sinusitis, and bronchiectasis. It is characterized with repeated lung infection and otitis media. In 50\% of Kartagener's syndrome cases, situs inversus exists (8.9). If respiratory problems in these cases are given the right treatment at the right time, problems due to situs inversus are not expected. But bronchiectasis signals can also develop in the early phase (10). In our case, a very detailed story was attained from the patient about additional diseases. Isolated situs inversus totalis was confirmed in the patient as there was no evidence of ciliary dysfunction. Situs anomalies are variable and especially in complex cases, before a more original diagnosis is made, good determination of anatomy is important. There are many diseases in differential diagnosis of patients who go to the emergency service with acute abdomen. In the presence of the SIT and intestinal malrotation, the abnormal position of the appendix makes the diagnosis difficult (11). Therefore, it is important in differential diagnosis not to forget about rare cases such as situs inversus totalis. In cases with few visits to health care institutions, and when the patients' story is not told to the physician the diagnosis gets difficult.

In physical differential examination, the right position of heart pulse, the significant intensity of the heart sounds in the right compared to the left side and palpability of the liver on the left suggest situs inversus. Dextrocardia in direct graphies and presence of the stomach fundus gas on the right are in favor of situs Totalis. Electrocardiography findings are helpful in the diagnosis of dextrocardia.

As a result, a complete physical examination must be done in situs inversus totalis cases for cardiac and other potential structural anomalies. In particular, attention must be paid to cardiovascular system, respiratory passage and spinal anomalies.

\section{References}

1. Nelson MJ, Pesola GR. Left lower quadrant pain of unusual cause. The Journal of Emergency Medicine 2001; 20: 241-245.

2. Kamiyama T, Fujiyoshi F, Hamada H, et al. Left sided acute appendicitis with intestinal malrotation. Radiation Medicine 2005; 23: 125-127.

3. Song JY, Rana N, Rottman CA, et al. Laparoscopic appendectomy in a female patient with situs inversus: Case report and literature review. JSLS. 2004; 8: $175-$ 177.

4. Neyzi O, Ertuğrul T. Pediatri 3. Edition. Nobel Tip Kitabevi İstanbul, 2002: 900-967.

5. Coban E, Duman A, Artac M, Dinçer D, Timuragaoglu A. Situs İnversus Totalis and akalazya: A case report. Gastroenterohepatoloji 2002; 13: 99102.

6. Altunhan H, Annagür A, Ors R. The assosiation of Carpenter Syndrom and Situs Inversus Totalis: First case report. Turkiye Klinikleri J Med Sci 2011; 31: 464-467.

East J Med Volume:23, Number:2, April-June/2018 
7. Ozgen B, Haliloglu M, Besim A. Jeune syndrome with Situs inversus totalis. Turk J Diagn Intervent Radiol 2001; 7: 592-594.

8. Kayhan A, Lakadamyali H, Oommen J, Oto A. Polysplenia syndrome accompanied with situs inversus totalis and annular pancreas in an elderly patient. Clin Imaging 2010; 34: 472-475.

9. Cowan MJ, Gladwin MT, Shelhamer JH. Disorders of ciliary motility. Am J Med Sciences 2001; 321: 3-10.
10. Kashif A, Masud M, Manzoor SM, Haneef S. Kartagener's Syndrome and Acute Appendicit. Ayub Med Coll Abbottabad 2010; 22: 176-177.

11. Brever RJ, Golden F, Hitch D, et al. Abdominal pain: an analysis of 1,000 consecutive cases in a university hospital emergency room. Am J Surg 1976; 131: 219223. 